\title{
Impacto dos Stents Farmacológicos no Tratamento Percutâneo de Lesões Coronárias em Bifurcação: Resultados Clínicos Tardios de um Estudo Comparativo Incluindo Pacientes do "Mundo-Real"
}

\author{
Alaor Mendes ${ }^{1}$, Amanda G. M. R. Sousa ${ }^{1,2}$, Ricardo A. Costa ${ }^{1,2}$, Adriana Moreira ${ }^{2}$, José Ribamar Costa Jr. ${ }^{1}$, \\ Galo Maldonado', Manuel N. Cano², André Lima', Rodolfo Staico', Fausto Feres', Luiz A. Mattos', \\ Alexandre Abizaid', Dimytri Siqueira', Otávio Berwanger², J. Eduardo Sousa ${ }^{1,2}$
}

\section{RESUMO}

Fundamentos: Historicamente, as intervenções coronárias percutâneas (ICP) em lesões de bifurcação associam-se com elevadas taxas de complicações, principalmente em decorrência dos resultados subótimos no ramo lateral (RL) e da necessidade de revascularização da lesão-alvo (RLA) durante seguimento tardio. Avaliamos o impacto dos stents farmacológicos (SF) na evolução clínica tardia de pacientes não-selecionados provenientes da prática clínica diária com lesão envolvendo bifurcação. Método: A partir de maio de 2002, 195 pacientes com lesão de bifurcação foram consecutivamente tratados em dois centros clínicos (grupo SF = 89 pacientes; grupo stent não-farmacológico [SNF] = 106), e o seguimento clínico foi completo até dois anos. Resultados: Os dois grupos apresentavam perfis clínicos semelhantes, incluindo média das idades de 63 anos, e $25 \%$ de diabetes melito. A maioria das lesões tinha comprometimento significativo dos dois ramos $(78 \%$ vs. $76 \% ; p=0,82)$, a estratégia de stent provisional RL foi utilizada na maioria dos casos $(75 \%$ vs. $89 \%$ de SNF; $\mathrm{p}=0,45$ ), e o sucesso angiográfico no RL (fluxo TIMI 3, estenose residual $<50 \%$, sem dissecção) foi atingido em $80 \%$ vs. $78 \%(p=0,56)$, nos grupos SF e SNF, respectivamente. Aos 24 meses de seguimento, o grupo SF apresentou maior sobrevida livre de RLA $(97,8 \%$ vs. $82,1 \%$; $p<0,0001)$, com conseqüente maior sobrevida livre de eventos cardíacos adversos maiores (ECAM) $(93,3 \%$ vs. $77,4 \% ; p=0,003)$. A trombose de stent ocorreu em 2 pacientes em cada grupo. Conclusões: Em pacientes nãoselecionados do "mundo-real", o uso de SF para o tratamento de lesões coronárias em bifurcação mostrou-se

\section{SUMMARY}

The Impact of Drug-eluting Stents in the Percutaneous Treatment of Bifurcation Coronary Lesions: Late Clinical Outcomes of a Comparative Study Including "Real-World" Patients

Background: Historically, percutaneous coronary interventions $(\mathrm{PCl})$ in bifurcation lesions are associated with high rates of complications, especially due to suboptimal results in the lateral branch (LB) and the need for target lesion revascularization (TLR) during long-term follow-up. We assess the impact of drug-eluting stents in the late clinical follow-up of non-selected patients with bifurcation lesions coming from the daily clinical practice. Methods: Since May, 2002, 195 patients with bifurcation lesions were treated consecutively at two clinical centers [drugeluting stent $(D E S)$ group $=89$; non drug-eluting stent (NDES) group $=106$ ] and followed up for two years. Results: The two groups presented similar clinical profiles including mean age of 63 years and $25 \%$ with diabetes mellitus. The majority of lesions had significant involvement of the two branches ( $78 \%$ vs. $76 \% ; p=0.82$ ), the provisional LB stent strategy was used in the majority of cases $(75 \%$ DES vs. $89 \%$ NDES; $p=0.45)$, and the angiographic success in the lateral branch (TIMI 3 flow, residual stenosis $<50 \%$ without dissection) was achieved in $80 \%$ vs. $78 \%$ $(p=0.56)$ in the DES and NDES groups, respectively. After 24 months of follow-up, the DES group showed a higher survival free of TLR $(97.8 \%$ vs. $82.1 \%$; $p<0.0001)$, resulting in higher survival free of major adverse cardiac events (MACE) $(93.3 \%$ vs. $77.4 \% ; p=0.003)$. Stent thrombosis

\footnotetext{
Instituto Dante Pazzanese de Cardiologia - São Paulo, SP.

Instituto de Ensino e Pesquisa, Hospital do Coração - Associação do Sanatório Sírio - São Paulo, SP.

Correspondência: Ricardo Alves Costa. Instituto Dante Pazzanese de Cardiologia. Av. Dr. Dante Pazzanese, 500 - Ibirapuera - São Paulo, SP - CEP 04012-909

E-mail: rcosta@dantepazzanese.org.br

Recebido em: 18/9/2009 • Aceito em: 16/11/2008
} 
significantemente superior ao uso de SNF no seguimento clínico tardio, em especial no que se refere à efetividade clínica em reduzir as taxas de RLA, com conseqüente aumento na sobrevida livre de ECAM, sem o comprometimento de sua segurança tardia.

DESCRITORES: Stents farmacológicos. Angioplastia transluminal percutânea coronária. Doença das coronárias/ terapia. Resultado de tratamento.

A S lesões coronárias envolvendo bifurcações são encontradas em cerca de $15 \%$ a $20 \%$ dos pacientes com doença coronária aterosclerótica obstrutiva'. Historicamente, as intervenções coronárias percutâneas (ICP) em bifurcações apresentam maiores índices de complicações, como dissecção e oclusão aguda, e menores taxas de sucesso do procedimento (por exemplo, estenose residual significativa, infarto do miocárdio) quando comparadas às intervenções em lesões não-bifurcadas ${ }^{2}$. Tais eventos adversos estão relacionados principalmente a complicações e resultados subótimos obtidos no ramo lateral (RL) das bifurcações, com destaque para as lesões envolvendo tanto o vaso principal (VP) como o RL, denominadas "lesões verdadeiras" de bifurcação ${ }^{3,4}$. Além disso, vários estudos prévios demonstraram pior prognóstico das ICP em lesões de bifurcação comparadas às lesões não-bifurcadas, incluindo elevadas taxas de reestenose (25\% a $50 \%$ ) e necessidade de revascularização da lesão-alvo (RLA) durante o seguimento clínico médio e tardio $(15 \%$ a $30 \%)^{5,6}$.

A introdução de stents farmacológicos (SF) revolucionou o tratamento percutâneo da doença coronária. Vários estudos randomizados comparando SF versus stents não-farmacológicos (SNF) no tratamento de lesões simples (não-bifurcadas) demonstraram benefício sustentado significativo a favor dos SF, principalmente pela marcante redução de reestenose e necessidade de $\mathrm{RLA}^{7,8}$. Notavelmente, pequenos estudos recentes comparando SF e SNF, em lesões complexas envolvendo bifurcações, sugeriram manutenção dos benefícios clínicos associados aos SF nesse subgrupo de alto risco, incluindo diminuição significativa de RLA no seguimento clínico a médio prazo ${ }^{9}$. No entanto, a evolução clínica a longo prazo de populações do "mundo-real" com lesões de bifurcação tratadas com SF permanece desconhecida. Logo, reportamos o impacto dos SF nos resultados clínicos tardios (dois anos) de pacientes não-selecionados provenientes da prática diária do "mundo-real" com lesão de bifurcação. occurred in 2 patients in each group. Conclusions: In nonselected patients from the "real-world", the use of DES to treat bifurcation coronary lesions was superior to the use of NDES stents in the long-term clinical follow-up, especially with respect to their clinical effectiveness in reducing the rates of TLR, with consequent increase in survival free from MACE, without compromising longterm safety.

DESCRIPTORS: Drug-eluting stents. Angioplasty, transluminal, percutaneous coronary. Coronary disease/therapy. Treatment outcome.

\section{MÉTODO}

\section{População do estudo}

Foram incluídos neste estudo pacientes com apenas uma lesão coronária envolvendo bifurcação, tratados com SF e incluídos no Registro DESIRE (DrugEluting Stent In the REal World), cujos detalhes do protocolo e os resultados clínicos globais já foram reportados previamente ${ }^{10}$. Em resumo, o Registro DESIRE é um estudo clínico prospectivo, não-randomizado, de braço único, com inclusão consecutiva de pacientes, realizado em uma instituição médica particular (Hospital do Coração - Associação do Sanatório Sírio, São Paulo, SP, Brasil), onde o SF tem sido utilizado como estratégia preferencial em pacientes encaminhados para ICP de rotina ou emergência desde maio de 2002 (inclusão em andamento). O Registro DESIRE tem como objetivo primário investigar a evolução clínica tardia de pacientes tratados com SF no cenário da prática diária do "mundo-real".

Para essa subanálise envolvendo apenas bifurcações coronárias, foram incluídos pacientes tratados a partir de maio de 2002, com seguimento clínico até dois anos, e esse subgrupo (grupo SF) foi comparado a uma coorte de pacientes não-selecionados com lesão coronária em bifurcação, e tratados consecutivamente com SNF (grupo SNF) em uma instituição médica pública (Instituto Dante Pazzanese de Cardiologia, São Paulo, SP, Brasil), a qual disponibilizava apenas o SNF como dispositivo de escolha para a ICP de emergência ou rotina durante o mesmo período de inclusão e seguimento (a partir de maio de 2002).

O estudo está em adequação com a Declaração de Helsinque relacionada à investigação em humanos e foi aprovado pelo Comitê de Ética das referidas instituições.

\section{Procedimento}

As ICP foram realizadas de acordo com as diretrizes vigentes, e a decisão final sobre a estratégia do procedimento ficou a critério do operador. 
Os seguintes SF foram utilizados no grupo SF: Cypher $^{\mathrm{TM}}$ - liberador de sirolimus (Cordis Corporation, Warren, NJ, Estados Unidos); Taxus ${ }^{\mathrm{TM}}$ - liberador de paclitaxel (Boston Scientific, Natick, MA, Estados Unido); Endeavor ${ }^{\mathrm{TM}}$ - liberador de zotarolimus (Medtronic, Santa Rosa, CA, Estados Unidos); e Xience ${ }^{T M} \mathrm{~V}$ - liberador de everolimus (Abbott Vascular, Abbott Park, IL, Estados Unidos).

Dentre os SNF utilizados no grupo SNF, encontramos: Liberté ${ }^{\mathrm{M}}$ (Boston Scientific, Natick, MA, Estados Unidos), Driver ${ }^{\mathrm{TM}}$ (Medtronic, Santa Rosa, CA, Estados Unidos), e Bx Sonic ${ }^{\mathrm{TM}}$ ou Bx Velocity ${ }^{\mathrm{TM}}$ (Cordis Corporation, Warren, NJ, Estados Unidos).

Ao operador coube decidir sobre o tipo de stent a ser implantado, bem como a estratégia final do procedimento, incluindo técnica com um ou dois stents, realização de pré-dilatação, pós-dilatação simples, e/ou pós-dilatação simultânea dos dois vasos da bifurcação (kissing-balloon), e também a utilização de inibidores de glicoproteína IIb/IIla intraprocedimento. O protocolo antitrombótico consistia na administração de dois agentes antiplaquetários: ácido acetilsalicílico (aspirina), na dose de $500 \mathrm{mg}, 24$ horas antes da ICP (se não utilizado previamente) e mantido indefinidamente (100 mg a 200 mg por dia), e um tienopiridínico, incluindo pré-tratamento com clopidogrel 300 mg a $600 \mathrm{mg}$ (ou ticlopidina $500 \mathrm{mg}$ ) realizado 24 horas antes da intervenção, em casos eletivos $\geq 6$ horas antes do procedimento e em caso de síndrome coronária aguda (SCA) sem supradesnivelamento do segmento ST (se não utilizado previamente). A manutenção do tienopiridínico obedeceu à seguinte recomendação: clopidogrel 75 mg por dia (ou ticlopidina $250 \mathrm{mg}$ duas vezes por dia), prescrito inicialmente por três a seis meses, e, a partir do final do ano de 2006, por período mínimo de um ano, de acordo com as novas recomendações do Food and Drug Administration (FDA) dos Estados Unidos, para os pacientes que receberam $\mathrm{SF}^{11}$.

Já para os pacientes que receberam SNF, as mesmas doses de manutenção de tienopiridínico supracitadas foram prescritas, mas por período mais curto (um mês). Durante o procedimento foi administrada heparina endovenosa $(70 \mathrm{U} / \mathrm{kg}$ a $100 \mathrm{U} / \mathrm{kg}$ ) para manter o tempo de coagulação ativado > 250 segundos (ou $>200$ segundos, no caso de administração concomitante de inibidor da glicoproteína IIb/IIla). Eletrocardiograma (ECG) com 12 derivações foi obtido de rotina, antes do procedimento, imediatamente após o procedimento e depois de 24 horas. Exames laboratoriais de rotina incluíram enzimas cardíacas (CPK e Ck-MB) pré-procedimento (< 24 horas), 18-24 horas pós-procedimento, e diariamente até a alta hospitalar em caso de alteração das mesmas.

\section{Análise angiográfica}

A análise angiográfica qualitativa incluiu a avaliação da distribuição de placa conforme a classificação preconizada por Medina et al. ${ }^{12}$, que definiu a presença de "lesões verdadeiras" de bifurcação quando havia obstrução significativa (> 50\% de estenose), tanto no VP como no RL.

Em relação à análise quantitativa, após a administração intracoronária de nitrato $(50 \mu \mathrm{g}$ a $200 \mu \mathrm{g})$, estudos angiográficos seriados foram obtidos, incluindo angiografias pré- e pós-procedimento. As análises de angiografia coronária quantitativa (ACQ) foram realizadas após o procedimento (off-line) por operadores independentes, utilizando-se um sistema de análise quantitativa com detecção semi-automática das bordas luminais (Medis Medical Imaging System, Leiden, Holanda). VP e RL foram analisados de forma independente. O diâmetro luminal mínimo (DLM) e o diâmetro de referência do vaso (DRV), obtidos pela média dos segmentos $5 \mathrm{~mm}$ a $10 \mathrm{~mm}$ proximal e distal da lesão-alvo no VP e $5 \mathrm{~mm}$ a $10 \mathrm{~mm}$ distal no RL, foram utilizados para calcular o diâmetro de estenose (DE):

$$
\mathrm{DE}=(1-\mathrm{DLM} / \mathrm{DRV}) \times 100 .
$$

\section{Objetivos, definições e seguimento clínico}

O objetivo primário desta análise foi a comparação da taxa de eventos cardíacos adversos maiores (ECAM) combinados, incluindo morte cardíaca, infarto do miocárdio (IAM) e RLA, entre os dois grupos de pacientes (SF vs. SNF) incluídos no período do estudo. Como desfecho secundário, compararam-se, isoladamente, as taxas de óbito cardíaco, IAM, RLA, e também trombose do stent. Como regra, todos os óbitos foram considerados cardíacos, a não ser que uma causa não-cardíaca pudesse ser claramente estabelecida por estudo clínico ou patológico. IAM foi classificado como com onda $Q$ e sem onda $Q$ (não-Q), sendo o diagnóstico baseado no aparecimento de nova onda Q patológica em duas ou mais derivações contíguas no ECG e/ou elevação da isoenzima Ck-MB > 3 vezes o limite superior da normalidade. A trombose de stent (TS) foi definida de acordo com as proposições do Academic Research Consortium (ARC) ${ }^{13}$ e classificada como: definitiva - presença de SCA e confirmação angiográfica ou anatomopatológica da oclusão do stent; provável - ocorrência de morte súbita $<30$ dias pós-procedimento índice ou IAM no território miocárdico tratado, sem confirmação angiográfica da oclusão do stent; e possível - ocorrência de morte súbita > 30 dias após o procedimento índice. Em relação à ocorrência temporal, a TS foi classificada como: aguda ( $\leq 24$ horas do procedimento), subaguda (ocorrência entre 24 horas e 30 dias pós-procedimento), tardia (ocorrência entre 1 e 12 meses pós-procedimento), e muito tardia (ocorrência > 12 meses pós-procedimento). O sucesso angiográfico foi definido como: fluxo TIMI grau 3, estenose residual < 50\% pela ACQ, e ausência de complicações agudas, como dissecção e oclusão em ambos os VP e RL ao final do procedimento. O sucesso do procedimento foi definido como 
Mendes A, et al. Impacto dos Stents Farmacológicos no Tratamento Percutâneo de Lesões Coronárias em Bifurcação: Resultados Clínicos Tardios de um Estudo Comparativo Incluindo Pacientes do "Mundo-Real". Rev Bras Cardiol Invas. 2008;16(4):406-414.

sucesso angiográfico mais ausência de ECAM na fase intra-hospitalar. O seguimento clínico foi realizado em 1, 6, e 12 meses, e anualmente até 5 anos após o procedimento, e consistiu de visita médica agendada ou contato telefônico, sendo realizado segundo protocolo predefinido. Para essa análise, foram incluídos apenas pacientes com seguimento clínico > 1 ano.

\section{Análise estatística}

Estimando-se taxa de ECAM de $25 \%$ entre os pacientes tratados com SNF e de $12,5 \%$ para os tratados com SF, um total de 85 pacientes para cada grupo nos daria poder estatístico de $90 \%$, com alfa de $5 \%$ e erro beta de $10 \%$ para demonstrar redução de $50 \%$ na incidência de ECAM com SF. As variáveis contínuas estão apresentadas como média \pm desvio padrão e foram comparadas pelo teste $t$ de Student não-pareado para coortes de distribuição normal. As variáveis categóricas estão reportadas como números absolutos e porcentagens (\%) e foram comparadas pelo teste do qui-quadrado de Pearson ou teste exato de Fisher, quando apropriado. As curvas de sobrevida livre de eventos no período médio de seguimento foram geradas pelo método de Kaplan-Meier e comparadas pelo teste de log-rank. Considerou-se estatisticamente significante um valor de $\mathrm{p}<0,05$ (análise bicaudal). As análises foram realizadas com o auxílio do programa estatístico SPSS versão 16.0 (SPSS Inc., Chicago, IL, Estados Unidos).

\section{RESULTADOS}

\section{Características clínicas basais, dados angiográficos e do procedimento}

Um total de 195 pacientes foi incluído nesta análise (SF 89; SNF 106), com média de tempo de seguimento clínico de 2 anos (Figura 1). A Tabela 1 apresenta as características clínicas basais. No geral, a média das idades era de 62 anos, um quarto dos pacientes tinha diabetes melito $(30,1 \%$ no grupo SNF vs. $20,9 \%$ no grupo SF; $p=0,52$ ), e cerca de $35 \%$ dos pacientes apresentavam-se com SCA na admissão (33,7\% vs. $36 \% ; p=0,68)$. As lesões envolviam a artéria descendente anterior e o ramo diagonal na maioria dos casos (74\% no grupo SF vs. $67 \%$ no grupo SNF; $p=0,38)$, e três quartos das lesões envolviam de forma significativa ambos os ramos da bifurcação. A Tabela 2 apresenta os dados angiográficos e do procedimento. O grupo de pacientes tratados com SNF utilizou mais a terapia com inibidor da glicoproteína Ilb/IIla intraprocedimento (14\% no grupo SNF vs. $6,5 \%$ no grupo $S F ; p=0,06)$. A estratégia preferencial entre os operadores de ambos os grupos foi a técnica de stent provisional no RL com implante de um stent no VP $(75 \%$ no grupo SF e $89 \%$ no grupo SNF; $p=0,45)$, terminando com kissing-balloon na maioria dos casos (64\% no grupo SF vs. $71 \%$ no grupo SNF; $p=0,64)$. O sucesso angiográfico pós-procedimento relaciona-

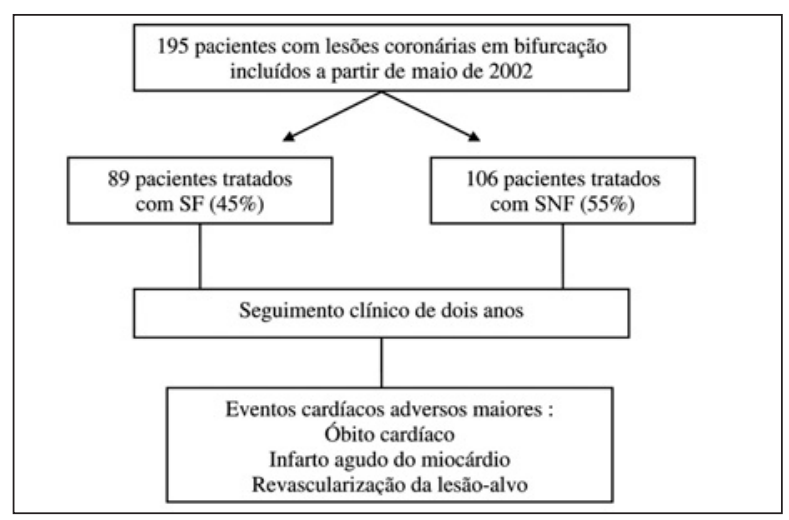

Figura 1 - Fluxograma de inclusão de pacientes. SF = stent farmacológico; SNF = stent não-farmacológico.

do ao VP foi atingido em 100\% dos casos em ambos os grupos, e resultados subótimos associados ao RL foram observados em cerca de $20 \%$ dos pacientes de cada coorte $(80 \%$ no grupo SF vs. $78 \%$ no grupo SNF; $\mathrm{p}=0,56)$.

A Tabela 3 apresenta os resultados da análise de ACQ.

\section{Eventos clínicos}

Na fase intra-hospitalar, não ocorreu morte cardíaca ou necessidade de nova intervenção na lesão-alvo em ambos os grupos; no entanto, 3,4\% dos pacientes do grupo SF e 4,7\% do grupo SNF evoluíram com IAM não-Q, sem diferença estatística detectável $(p=0,58)$. No seguimento clínico tardio, a incidência acumulada de ECAM foi significantemente menor entre os pacientes tratados com SF (7,8\% vs. 24,5\%; $p=0,02)$, uma vez que os SF reduziram de maneira absoluta a freqüência de RLA $(2,2 \%$ vs. 18,0\%; $p<0,001)$. As taxas de mortalidade cardíaca e a freqüência de IAM ao longo do seguimento clínico foram baixas e similares nos grupos SF e SNF $(2,2 \%$ vs. 1,9\%, p = 0,85; e 1,1\% vs. $1,0 \%, p=N A$, respectivamente) (Tabela 4 ). Durante o período de seguimento, 2 pacientes de cada coorte evoluíram com TS. Ao fim de 24 meses de evolução clínica, 97,8\% dos pacientes tratados com SF e $82,1 \%$ dos tratados com SNF encontravam-se livres da necessidade de RLA (teste log-rank, risco relativo $[R R]=23,4$; intervalo de confiança de 95\% [IC 95\%]: $22,6-24,2 ; p<0,0001)$, enquanto $93,3 \%$ dos pacientes do grupo SF e $77,4 \%$ do grupo SNF evoluíram livres de qualquer ECAM (teste log-rank; RR = 22,5; IC 95\%: 21,5-23,4; $\mathrm{p}=0,003)$, conforme ilustrado na Figura 2.

\section{DISCUSSÃO}

Estudos prévios comparando SF e SNF nos diferentes cenários de complexidade estimularam os investigadores a confirmar a superioridade desses dispositivos eluidores de fármacos no tratamento de um 
TABELA 1

Características clínicas basais

\begin{tabular}{lccc}
\hline Variável & SF $\mathbf{( n = 8 9 )}$ & SNF $(\mathbf{n}=\mathbf{1 0 6})$ & Valor de $\mathbf{p}$ \\
\hline Média das idades, anos & $62,1 \pm 11,1$ & $62,9 \pm 9,1$ & 0,85 \\
Sexo masculino, \% & 71,2 & 64,8 & 0,56 \\
Diabetes melito, \% & 20,9 & 30,1 & 0,52 \\
Hipertensão, \% & 70,8 & 82,5 & 0,46 \\
Dislipidemia, \% & 63,7 & 75,1 & 0,41 \\
Tabagismo atual, \% & 29,8 & 35,4 & 0,53 \\
História familiar de DAC, \% & 53,2 & 30,4 & 0,37 \\
IAM prévio, \% & 17,9 & 19,1 & 0,51 \\
ICP prévia, \% & 24,7 & 21,3 & 0,62 \\
CRM prévia, \% & 17,7 & 15,8 & 0,51 \\
AVE prévio, \% & 1,1 & 2,1 & 0,34 \\
História de DVP, \% & 2,2 & 4,0 & 0,28 \\
Insuficiência renal", \% & 12,8 & 14,6 & 0,51 \\
Apresentação clínica, \% & & \\
$\quad$ Angina estável & 32,5 & 29,1 & 0,26 \\
Angina instável & 22,4 & 25,3 & 0,25 \\
IAM & 11,3 & 10,7 & 0,42 \\
Isquemia silenciosa & 33,8 & 34,9 & 0,36 \\
\hline
\end{tabular}

Valores são expressos como freqüência ou média \pm desvio padrão. " Definida como creatinina sérica basal $\geq 1,5 \mathrm{mg} / \mathrm{dl}$. AVE $=$ acidente vascular encefálico; CRM = cirurgia de revascularização do miocárdio; DAC = doença arterial coronária; DVP = doença vascular periférica; IAM = infarto agudo do miocárdio; ICP = intervenção coronária percutânea; $\mathrm{n}=$ número de pacientes; SF = stent farmacológico; SNF = stent não-farmacológico.

subgrupo de pacientes portadores de lesões coronárias em bifurcação, uma vez que os SF promoviam marcante redução na hiperplasia neo-intimal e conseqüente redução da necessidade de RLA a longo prazo ${ }^{14}$.

Com o objetivo de aferir a exeqüibilidade e a segurança do uso dos SF no tratamento de lesões em bifurcação, Thuesen et al. ${ }^{15}$ reportaram uma subanálise do estudo randomizado SCANDSTENT comparando SF e SNF no tratamento de lesões coronárias em bifurcação durante seguimento clínico de sete meses, incluindo amostra com $54 \%$ de pacientes portadores de lesão significativa, tanto no VP como no RL, e que foram abordadas, nesses casos de "bifurcação verdadeira", pelas técnicas de dois stents (aproximadamente 50\% dos casos) em ambos os grupos. A superioridade e a segurança tardia dos SF nesse complexo subgrupo de pacientes e lesões foram confirmadas pela redução das taxas de RLA $(6,0 \%$ vs. $21,1 \% ; p=0,016)$, sobrevida livre de ECAM $(91,2 \%$ vs. $71,9 \%$; teste logrank; $\mathrm{p}=0,006)$, e índices de TS (0\% vs. 9,0\%; $\mathrm{p}=0,02)$ aos sete meses de acompanhamento clínico. Resultados semelhantes foram encontrados em nosso estudo, em que também os SF apresentaram menor incidência acumulada de ECAM relacionada à redução absoluta da freqüência de RLA $(2,2 \%$ vs. $18,0 \%$; p < 0,001) e maior sobrevida livre de qualquer ECAM ao final de dois anos (Figura 2B). Em nossa série, a maioria dos pacientes foi tratada com apenas um stent (cerca de $80 \%$ dos casos), apesar de grande parte das lesões envolver de forma significativa os dois ramos da bifurcação (cerca de $80 \%$ de "lesões verdadeiras de bifurcação"). Uma série de estudos randomizados prévios comparou o uso de um stent ao uso de dois stents na abordagem técnica de lesões coronárias em bifurcação. Em pesquisas realizadas por Pan et al. ${ }^{16}$ e Colombo et al. ${ }^{17}, 303$ pacientes com lesões em bifurcação foram randomizados (1:1) para técnica provisional com implante de um stent versus estratégia complexa com implante de dois stents (sendo a técnica T-stenting preferencial entre os operadores em 95\% dos casos), e não foi observada diferença significativa entre os grupos de um stent versus dois stents quando foram analisados os índices de sucesso imediato do procedimento (90,3\% vs. 91,1\%; p = NS) e sobrevida livre dos eventos combinados de morte cardíaca, IAM e RLA aos 18 meses de seguimento (81,1\% vs. 76,7\%; $\mathrm{p}=\mathrm{NS}$ ). Nesses estudos randomizados com pacientes provenientes de diversos centros e tratados com stents eluídos por sirolimus ou paclitaxel, as duas técnicas (um stent versus dois stents) apresentaram equivalência clínica; no entanto, observou-se taxa de cross-over para a técnica com implante de dois stents na série reportada por Colombo et al. ${ }^{17}$ em até $25 \%$ dos casos, e a população estudada por Pan et al. ${ }^{16}$ apresentava 
TABELA 2

Dados angiográficos e do procedimento

\begin{tabular}{|c|c|c|c|}
\hline Variável & $\mathrm{SF}(\mathbf{n}=\mathbf{8 9})$ & SNF $(n=106)$ & Valor de $p$ \\
\hline \multicolumn{4}{|l|}{ Morfologia da lesão } \\
\hline "Lesão verdadeira" de bifurcação*, \% & 78 & 76 & 0,82 \\
\hline \multicolumn{4}{|l|}{ Classificação de Medina, \% } \\
\hline $1,1,1$ & 32 & 31 & 0,68 \\
\hline $1,0,1$ & 15 & 16 & 0,60 \\
\hline $0,1,1$ & 31 & 29 & 0,54 \\
\hline Calcificação moderada/grave (VP/RL), \% & 28 & 30 & 0,62 \\
\hline Excentricidade (VP), \% & 26 & 22 & 0,45 \\
\hline Angulação > 45 graus da bifurcação, \% & 4,8 & 5,1 & NA \\
\hline \multicolumn{4}{|l|}{ Procedimento } \\
\hline \multicolumn{4}{|l|}{ Pré-dilatação, \% } \\
\hline VP & 64 & 66 & 0,64 \\
\hline$R L$ & 50 & 47 & 0,60 \\
\hline \multicolumn{4}{|l|}{ Pós-dilatação simples, \% } \\
\hline VP & 24 & 19 & 0,51 \\
\hline$R L$ & 12 & 10 & 0,60 \\
\hline Pós-dilatação final com kissing-balloon, \% & 64 & 71 & 0,64 \\
\hline Técnica com stent único, \% & 75 & 89 & 0,45 \\
\hline Técnica com implante de dois stents, \% & 25 & 11 & 0,44 \\
\hline \multicolumn{4}{|l|}{ Sucesso angiográfico, \% } \\
\hline VP & 100 & 100 & NA \\
\hline $\mathrm{RL}$ & 80 & 78 & 0,56 \\
\hline
\end{tabular}

Valores são expressos como frequiência ou média \pm desvio padrão. ${ }^{*}$ Definida como estenose $>50 \%$ tanto no VP como no RL. $n=$ número de pacientes; NA = não aplicável; RL = ramo lateral; SF = stent farmacológico; SNF = stent não-farmacológico; VP = vaso principal.

TABELA 3

Angiografia coronária quantitativa

\begin{tabular}{|c|c|c|c|c|c|c|}
\hline \multirow[b]{2}{*}{ Variável } & \multicolumn{3}{|c|}{ Pré-procedimento } & \multicolumn{3}{|c|}{ Pós-procedimento } \\
\hline & SF $(n=89)$ & SNF $(n=106)$ & Valor de $p$ & SF $(n=89)$ & SNF $(n=106)$ & Valor de $p$ \\
\hline \multicolumn{7}{|c|}{ Extensão da lesão, mm } \\
\hline VP & $20,15 \pm 6,00$ & $13,48 \pm 4,45$ & 0,48 & - & - & - \\
\hline $\mathrm{RL}$ & $6,52 \pm 2,33$ & $5,47 \pm 3,42$ & 0,61 & - & - & - \\
\hline \multicolumn{7}{|c|}{$\mathrm{DRV}, \mathrm{mm}$} \\
\hline VP & $2,81 \pm 0,32$ & $3,10 \pm 0,42$ & 0,55 & - & - & - \\
\hline $\mathrm{RL}$ & $1,85 \pm 0,53$ & $2,08 \pm 0,46$ & 0,81 & - & - & - \\
\hline \multicolumn{7}{|c|}{$\mathrm{DLM}, \mathrm{mm}$} \\
\hline VP & $1,04 \pm 1,32$ & $1,08 \pm 0,35$ & NA & $2,85 \pm 0,22$ & $2,82 \pm 0,55$ & NA \\
\hline$R L$ & $0,79 \pm 0,46$ & $0,86 \pm 0,54$ & $>0,84$ & $1,56 \pm 0,57$ & $1,53 \pm 0,43$ & NA \\
\hline \multicolumn{7}{|l|}{$\mathrm{DE}, \%$} \\
\hline VP & $67,52 \pm 14,16$ & $69,11 \pm 8,82$ & 0,64 & $5,32 \pm 2,91$ & $10,68 \pm 5,62$ & 0,18 \\
\hline $\mathrm{RL}$ & $50,94 \pm 13,48$ & $52,61 \pm 12,84$ & 0,62 & $24,15 \pm 13,72$ & $27,02 \pm 17,9$ & 0,55 \\
\hline
\end{tabular}

Valores são expressos como freqüência ou média \pm desvio padrão. DE = diâmetro de estenose; DLM = diâmetro luminal mínimo; $\mathrm{DRV}=$ diâmetro de referência do vaso; $\mathrm{n}=$ número de pacientes; $\mathrm{SF}=$ stent farmacológico; $\mathrm{SNF}=$ stent $\mathrm{não}-\mathrm{farmacológico}$; $\mathrm{VP}=$ vaso principal; RL = ramo lateral. 
TABELA 4

Resultados clínicos

\begin{tabular}{|c|c|c|c|c|c|c|}
\hline \multirow[b]{2}{*}{ Variável } & \multicolumn{3}{|c|}{ Fase hospitalar } & \multicolumn{3}{|c|}{ Seguimento clínico tardio } \\
\hline & SF $(n=89)$ & SNF $(n=106)$ & Valor de $p$ & SF $(n=89)$ & SNF $(n=106)$ & Valor de $p$ \\
\hline ECAM, \% & $3,4 \%$ & $4,7 \%$ & 0,58 & $7,8 \%$ & $24,5 \%$ & 0,02 \\
\hline Óbito cardíaco, \% & $0 \%$ & $0 \%$ & NA & $2,2 \%$ & $1,9 \%$ & 0,85 \\
\hline IAM sem onda $Q, \%$ & $3,4 \%$ & $4,7 \%$ & 0,58 & $3,4 \%$ & $5,7 \%$ & 0,40 \\
\hline IAM com onda Q, \% & $0 \%$ & $0 \%$ & NA & $1,1 \%$ & $1,0 \%$ & NA \\
\hline RLA, \% & $0 \%$ & $0 \%$ & NA & $2,2 \%$ & $18,0 \%$ & $<0,001$ \\
\hline Trombose de stent, \% & $0 \%$ & $0 \%$ & NA & $2,2 \%$ & $1,9 \%$ & 0,85 \\
\hline
\end{tabular}

Valores são expressos como freqüência ou média \pm desvio padrão. ECAM = eventos cardíacos adversos maiores; IAM = infarto do miocárdio; $\mathrm{n}=$ número de pacientes; NA = não aplicável; RLA = revascularização da lesão-alvo; SF = stent farmacológico; SNF = stent não-farmacológico.

A

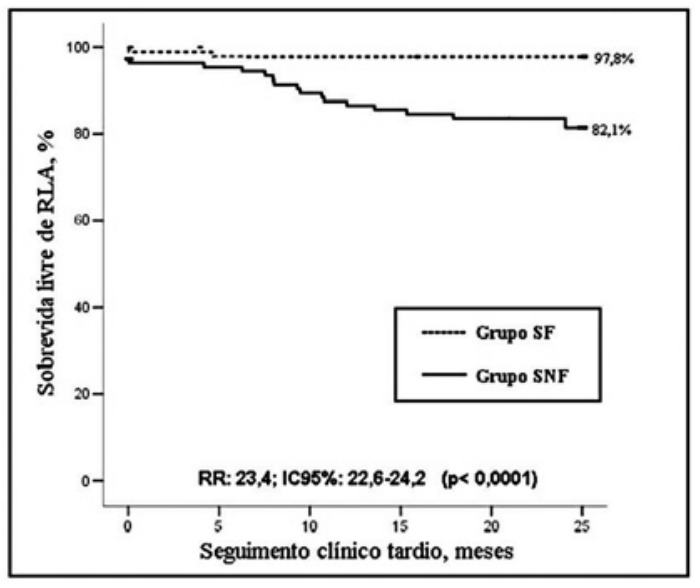

B

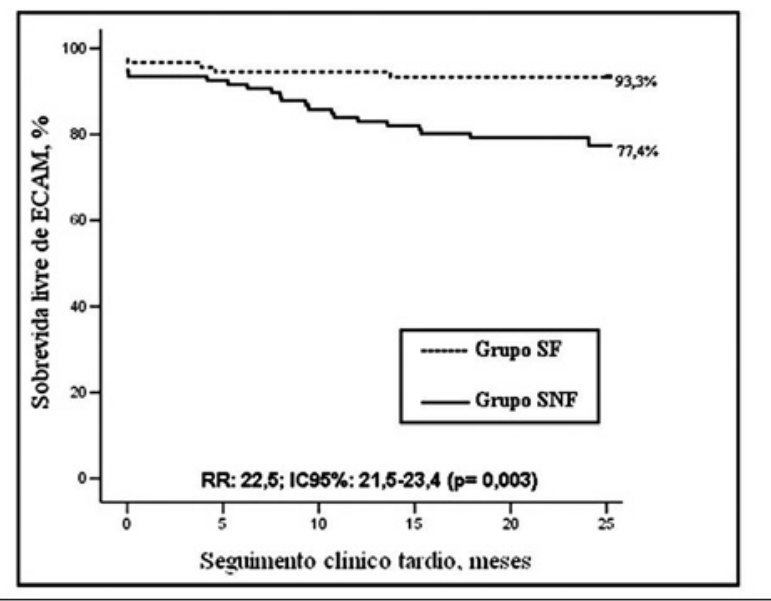

Figura 2 - Curva de sobrevida livre de eventos de Kaplan-Meier comparando pacientes tratados com stents farmacológicos e stents nãofarmacológicos. A. Curva de sobrevida livre de revascularização da lesão-alvo. B. Curva de sobrevida livre de eventos cardíacos adversos maiores. $\mathrm{ECAM}=$ eventos cardíacos adversos maiores; RLA = revascularização da lesão-alvo; SF = stent farmacológico; SNF = stent não-farmacológico; $\mathrm{RR}=$ risco relativo; $\mathrm{IC}=$ intervalo de confiança.

características angiográficas basais de menor complexidade (por exemplo, "lesões não-verdadeiras de bifurcação", RL de menor calibre e importância, e lesões curtas no VP). Após a publicação do estudo Nordic Bifurcation $^{18}$, que randomizou 413 pacientes com lesões coronárias em bifurcação (sendo apenas 50\% "lesões verdadeiras de bifurcação"), a técnica provisional com implante de um stent tem sido considerada por muitos como alternativa inicial na abordagem de bifurcações, pois a estratégia com implante de dois stents não se associou a menores taxas de eventos cardíacos adversos durante seguimento clínico de seis meses $(2,9 \%$ vs. $3,4 \% ; p=N S)$, além de ter necessitado maiores tempos de fluoroscopia e quantidade de contraste. Recentemente, Jensen et al. ${ }^{19}$ (Nordic-Baltic PCI Study Group) publicaram os resultados clínicos de 14 meses do estudo Nordic Bifurcation, corroborando a equivalência entre as técnicas de um stent versus dois stents no que tange à redução de $\operatorname{ECAM}(9,5 \%$ vs. $8,2 \% ; p=N S)$, e também a segurança tardia dos SF expressa pelas taxas de TS $(2,5 \%$ vs. $0,5 \%$; $p=N S)$. Nessa mesma linha de investigação, Colombo ${ }^{20}$ apresentou os resultados do recente estudo CACTUS após trinta dias de acompanhamento clínico, e, segundo randomização (técnica provisional com implante de um stent versus as técnicas de 2 stents: T-stenting ou Crush) de aproximadamente 250 pacientes com 95\% de "lesões verdadeiras de bifurcação", mais uma vez o uso rotineiro de dois stents para o tratamento de bifurcação não apresentou superioridade angiográfica ou clínica e/ou diferença quanto à segurança tardia. No entanto, foi observada taxa de cross-over para a técnica de dois stents em até 30\% dos casos randomizados para a estratégia provisional com implante de 
Mendes A, et al. Impacto dos Stents Farmacológicos no Tratamento Percutâneo de Lesões Coronárias em Bifurcação: Resultados Clínicos Tardios de um Estudo Comparativo Incluindo Pacientes do "Mundo-Real". Rev Bras Cardiol Invas. 2008;16(4):406-414.

um stent no VP, mas sem diferença significativa nas taxas de TS. Dados preliminares de um estudo recente (INSIDE I) reportando ICP em bifurcações com guia de ultra-som intracoronário (USIC) sugerem que as lesões de bifurcação verdadeiras que se estendem para RLs mais calibrosos são as que mais se beneficiariam de uma estratégia com implante de dois stents ${ }^{21}$. Logo, atualmente, acredita-se que nas lesões de bifurcação com RL sem obstrução importante ou com estenose focal (ostial), a qual se apresenta responsiva à dilatação com cateter-balão, o tratamento com estratégia de stent provisional no RL seja indicado. Já as lesões com maior complexidade podem se beneficiar do tratamento com dois stents; no entanto, a melhor abordagem técnica ideal permanece controversa ${ }^{22}$.

As duas TSs observadas no grupo SF foram classificadas como definitivas (com confirmação angiográfica), e ocorreram em pacientes tratados com dois stents, sendo uma tardia (aproximadamente quatro meses) e outra muito tardia ( $>$ um ano), e ambas foram associadas ao quadro clínico de IAM com onda Q, seguido de RLA por cirurgia de revascularização do miocárdio. No entanto, as duas TSs do grupo SNF (também em pacientes tratados com dois stents) foram classificadas como possíveis, muito tardias (> um ano) e relacionadas ao óbito cardíaco. Vários estudos têm demonstrado aumento das taxas de TS com dois stents $(1,5 \%$ a $6 \%)$. As possíveis explicações incluem: lesões de maior complexidade, alta concentração de metal na "carina" da bifurcação, além de causas mecanísticas (subexpansão do stent, aposição incompleta das hastes do stent) e técnicas ${ }^{23}$. Em um estudo conduzido por Hoye et al. ${ }^{24}$, pacientes consecutivos tratados por técnica de crush (sirolimus [ $n=144]$ ou paclitaxel [n = 104]) apresentaram taxa de TS global de 4,3\% até o seguimento tardio. Notavelmente, a otimização técnica parece ser crítica para melhorar os resultados com dois stents. Ge et al. ${ }^{25}$ mostraram melhores resultados clínicos com a realização sistemática de kissingballoon. Também, um estudo com USIC pós-procedimento em pacientes tratados com crush demonstrou subexpansão sistemática do óstio do $R L$, a qual não era evidenciada pela angiografia, e aposição incompleta das hastes do stent na altura da "carina" em 60\% dos $\operatorname{casos}^{26}$. Assim, quando se utilizar técnica com dois stents, é fundamental a realização de pós-dilatação simples a altas pressões nos dois ramos e kissingballoon final para otimização dos resultados. Digno de nota é o fato de que os pacientes em nossa série tratados pela técnica de dois stents apresentaram relação direta com o incremento na incidência de TS; no entanto, não houve comprometimento de sua segurança tardia com SF ( $p=N S)$.

\section{Limitações do estudo}

O estudo inclui pacientes consecutivos de instituições clínicas de perfil heterogêneo, mas tratados pelo mesmo corpo clínico; logo, não esperamos viés de seleção significativo na análise, a não ser aquele relacionado à abordagem técnica individualizada de cada operador. A comparação entre os dois dispositivos (SF versus SNF) foi realizada de modo nãorandomizado, incluindo um grupo (SF) proveniente de uma subanálise de um registro realizado em centro único (Registro DESIRE). Mesmo assim, a análise das variáveis basais demonstrou semelhança no perfil clínico e angiográfico entre os dois grupos.

\section{CONCLUSÕES}

No presente estudo com inclusão de pacientes não-selecionados do "mundo real", o uso de SF para o tratamento de lesões coronárias em bifurcação mostrou-se significantemente superior ao uso de SNF, em especial no que se refere à efetividade clínica em reduzir as taxas de nova intervenção na lesão-alvo, com conseqüente aumento na sobrevida livre de ECAM sem o comprometimento de sua segurança tardia.

\section{AGRADECIMENTOS}

Os autores gostariam de reconhecer a dedicação e significativa colaboração dos seguintes profissionais envolvidos nesta análise: Cantídio Campos, Fábio Kawasetsu, Reginaldo Barreto, Valéria Ranieri e Wagner Pinto.

\section{REFERÊNCIAS BIBLIOGRÁFICAS}

1. Zack PM, Ischinger T. Experience with a technique for coronary angioplasty of bifurcational lesions. Cathet Cardiovasc Diagn. 1984;10(5):433-43.

2. Thomas M, Hildick-Smith D, Louvard Y, Albiero R, Darremont O, Stankovic G, et al. Percutaneous coronary intervention for bifurcation disease. A consensus view from the first meeting of European Bifurcation Club. Eurointerv. 2006;2:149-53.

3. Meier B, Gruentzig AR, King SB $3^{\text {rd }}$, Douglas JS Jr, Hollman $\mathrm{J}$, Ischinger $\mathrm{T}$, et al. Risk of side branch occlusion during coronary angioplasty. Am J Cardiol. 1984;53(1):10-4.

4. Cho GY, Lee CW, Hong MK, Kim JJ, Park SW, Park SJ. Effects of stent design on side branch occlusion after coronary stent placement. Catheter Cardiovasc Interv. 2001;52(1): 18-23.

5. Aliabadi D, Tilli FV, Bowers TR, Benzuly KH, Safian RD, Goldstein JA, et al. Incidence and angiographic predictors of side branch occlusion following high-pressure intracoronary stenting. Am J Cardiol. 1997;80(8):994-7.

6. Lefèvre $T$, Louvard $Y$, Morice MC, Dumas P, Loubeyre C, Benslimane $A$, et al. Stenting of bifurcation lesions: classification, treatments, and results. Catheter Cardiovasc Interv. 2000;49(3):274-83.

7. Moses JW, Leon MB, Popma JJ, Fitzgerald PJ, Holmes DR, O'Shaughnessy C, et al.; SIRIUS Investigators. Sirolimuseluting stents versus standard stents in patients with stenosis in a native coronary artery. N Engl J Med. 2003;349(14): 1315-23.

8. Lagerqvist B, James SK, Stenestrand U, Lindbäck J, Nilsson T, Wallentin L. Long-term outcomes with drug-eluting stents versus bare-metal stents in Sweden. N Engl J Med. 2007; 356(10):1009-19. 
Mendes A, et al. Impacto dos Stents Farmacológicos no Tratamento Percutâneo de Lesões Coronárias em Bifurcação: Resultados Clínicos Tardios de um Estudo Comparativo Incluindo Pacientes do "Mundo-Real". Rev Bras Cardiol Invas. 2008;16(4):406-414.

9. Pan M, Suárez de Lezo J, Medina A, Romero M, Hernández E, Segura J, et al. Simple and complex stent strategies for bifurcated coronary arterial stenosis involving the side branch origin. Am J Cardiol. 1999;83(9):1320-5.

10. Sousa AGMR, Costa Jr JR, Moreira A, Costa RA, Cano MN, Andrade GAM, et al. Evolução clínica tardia dos stents farmacológicos. Segurança e eficácia até cinco anos do registro DESIRE. Rev Bras Cardiol Invas. 2007;15(3):221-7.

11. Grines CL, Bonow RO, Casey DE Jr, Gardner TJ, Lockhart PB, Moliterno DJ, et al. Prevention of premature discontinuation of dual antiplatelet therapy in patients with coronary artery stents: a science advisory from the American Heart Association, American College of Cardiology, Society for Cardiovascular Angiography and Interventions, American College of Surgeons, and American Dental Association, with representation from the American College of Physicians. Circulation. 2007;115(6):813-8.

12. Medina A, Suárez de Lezo J, Pan M. A new classification of coronary bifurcation lesions. Rev Esp Cardiol. 2006;59(2): 183-4.

13. Serruys PW, Daemen J. Are drug-eluting stents associated with a higher rate of late thrombosis than bare metal stents? Late stent thrombosis: a nuisance in both bare metal and drug-eluting stents. Circulation. 2007;115(11):1433-9.

14. Marzocchi A, Saia F, Piovaccari G, Manari A, Aurier E, Benassi A, et al. Long-term safety and efficacy of drug-eluting stents: two-year results of the REAL (REgistro AngiopLastiche dell'Emilia Romagna) multicenter registry. Circulation. 2007; 115(25):3181-8.

15. Thuesen L, Kelbaek H, Kløvgaard L, Helqvist S, Jørgensen E, Aljabbari S, et al.; SCANDSTENT Investigators. Comparison of sirolimus-eluting and bare metal stents in coronary bifurcation lesions: subgroup analysis of the Stenting Coronary Arteries in Non-Stress/Benestent Disease Trial (SCANDSTENT). Am Heart J. 2006;152(6):1140-5.

16. Pan M, Suárez de Lezo J, Medina A, Romero M, Segura J, Ramírez A, et al. A stepwise strategy for the stent treatment of bifurcated coronary lesions. Catheter Cardiovasc Interv. $2002 ; 55(1): 50-7$.
17. Colombo A, Moses JW, Morice MC, Ludwig J, Holmes DR Jr, Spanos V, et al. Randomized study to evaluate sirolimuseluting stents implanted at coronary bifurcation lesions. Circulation. 2004;109(10):1244-9.

18. Steigen TK, Maeng M, Wiseth R, Erglis A, Kumsars I, Narbute I, et al. Randomized study on simple versus complex stenting of coronary artery bifurcation lesions: the Nordic bifurcation study. Circulation. 2006;114(18):1955-61.

19. Jensen J, Galloe A, Lassen J, Erglis A, Kumsars I, Steigen T, et al. Safety in simple versus complex stenting of coronary artery bifurcation lesions. The Nordic Bifurcation Study 14month follow-up results. Eurolntervention. 2008;4(2):229-33.

20. Colombo A. CACTUS: a prospective randomized comparison of crush versus provisional T-stenting in bifurcated coronary lesions: angiographic and 30-days result. In: TCT 2007 Late Breaking Clinical Trials, Washington; 2007.

21. Costa R, Furuichi S, Lansky A, et al. Midterm clinical outcomes from the intravascular ultrasound investigation of the parent vessel and the side branch in coronary bifurcation lesions treated with different stenting techniques (INSIDE I) trial. J Am Coll Cardiol. J Am Coll Cardiol. 2008;51 Suppl B:B51.

22. Latib A, Colombo A. Bifurcation disease: what do we know, what should we do? J Am Coll Cardiol Intv. 2008;1(3):218-26.

23. Costa RA, Moussa ID. Percutaneous treatment of coronary bifurcation lesions in the era of drug-eluting stents. Minerva Cardioangiol. 2006;54(5):577-89.

24. Hoye A, lakovou I, Ge L, van Mieghem CA, Ong AT, Cosgrave J, et al. Long-term outcomes after stenting of bifurcation lesions with the "crush" technique: predictors of an adverse outcome. J Am Coll Cardiol. 2006;47(10):1949-58.

25. Ge L, Airoldi F, lakovou I, Cosgrave J, Michev I, Sangiorgi GM, et al. Clinical and angiographic outcome after implantation of drug-eluting stents in bifurcation lesions with the crush stent technique: importance of final kissing balloon post-dilation. J Am Coll Cardiol. 2005;46(4):613-20.

26. Costa RA, Mintz GS, Carlier SG, Lansky AJ, Moussa I, Fujii $\mathrm{K}$, et al. Bifurcation coronary lesions treated with the "crush" technique: an intravascular ultrasound analysis. J Am Coll Cardiol. 2005;46(4):599-605. 